\title{
THE PROPERTIES OF MEIOTIC GENE CONVERSION IMPORTANT IN ITS EFFECTS ON EVOLUTION
}

\author{
B. C. LAMB \\ Department of Pure and Applied Biology, Imperial College, London SW7 2BB, England \\ Received 22.viii.83
}

\section{SUMMARY}

In order to determine how important gene conversion is as a force in populations, extensive surveys have been made to determine the limits, distributions and typical values of evolution-related conversion parameters from various fungi, Drosophila and maize. The conversion frequency, $c$, had an enormous range for different mutations within a species, with loci often having different means and limits for $c$. $b$, the frequency of a particular allelle in the products of meiotic tetrads or octads with aberrant segregation ratios, often showed values near the theoretical extremes; $d$, disparity in direction of conversion, was frequent and often extreme. $b$ and $d$ could only be studied in fungi, and their less extreme values in Saccharomyces than in Sordaria or Ascobolus are clearly related to the lack of frame-shift mutations in the yeast data. For different mutations at a locus, neither $c$ nor $b$ showed normal distributions; both gave dispersed distributions, sometimes multi-modal for $b ; c$ and $b$ were not usually correlated. $y$, the force of meiotic gene conversion on allele frequencies, had a large range of positive and negative values, with different loci often having quite different limits and mean absolute values. For different mutations at a locus, $y$ showed dispersed distributions, with little tendency to cluster around zero. The high proportion of non-zero $y$ values in all fungi where this could be studied, with large departures from zero being most extreme in Ascobolus and Sordaria brevicollis, shows that gene conversion could often be an important force in changing allele frequencies in favour of mutant or wild-type in these organisms. Even in organisms with much lower $c$ or absolute $d$ values, conversion could still be important, depending on dominance, selection coefficients and mutation rates. Presumed frame-shifts tended to have more extreme disparity and hence higher absolute $y$ values than did presumed base-substitutions, but with no consistent $c$ differences. Induced mutations had similar or slightly higher absolute $y$ values compared with spontaneous mutations.

\section{INTRODUCTION}

Gene conversion is increasingly considered important in evolution, in two respects. The one looked at here is the evolutionary effects of meiotic gene conversion between alleles in heterozygotes, involving normal meiotic pairing of homologous chromosomes. The other is the effects of intrachromosomal and interchromosomal gene conversion on the maintenance of sequence homogeneity within families of repeated eukaryotic genes, and the bringing about of concerted evolutionary changes in populations, with the proposed events not being related to homologous meiotic pairing (e.g., Klein and Petes, 1981; Dover et al., 1982; Dover, 1982; Mikus and Petes, 1982).

It has been established that meiotic gene conversion could be a significant process in evolution (Gutz and Leslie, 1976; Lamb, 1981; Lamb and Helmi, 1982), but the assessment of how important it actually is depends absolutely 
on the quantitative values of gene conversion parameters in relation to the other evolutionary factors, especially selection, drift and mutation rates. This paper surveys observed values from a range of organisms for the crucial conversion parameters, conversion frequency and the amount of disparity in the direction of conversion, for heterozygous sites. These results are also of interest for recombination mechanisms.

The organisms surveyed include eight genera of fungi, and Drosophila and Zea. Values are considered at three levels: single mutations under different conditions of environment or genotype; different mutations at a locus under the same or similar conditions, and all suitable mutations in a species, whatever the locus. Results are compared for different organisms, for frame-shifts and base-substitutions, and for spontaneous and induced mutations.

\section{DeFINITIONS}

These follow Lamb and Helmi (1982); $c$ is the meiotic gene conversion frequency, that is, the fraction of meioses giving aberrant segregation ratios from gene conversion at a heterozygous site; $b$ is the frequency of a particular allele (wild-type ( + ) for $+/ m$ (mutant), of $A$ for $A / a$ ) in meiotic tetrads or octads with aberrant segregation ratios; $d$ is the disparity in direction of conversion, where $d=b-0.5$. The force of gene conversion on allele frequencies is $y$, where $y=c(b-0 \cdot 5)$, i.e., the product of conversion frequency and disparity. With no disparity, $b=0.5, d=0, y=0$, conversion to $+($ or $A$ ) is as frequent as conversion to $m$ (or $a$ ). With more conversion to + than to $m, b>0.5, y$ and $d$ have positive values; with more conversion to $m$ than to $+, b<0 \cdot 5, y$ and $d$ have negative values. Tetrad or octad allele segregation ratios are given as $+: m$, so $6: 2$ is an octad with $6+$ spores, $2 m$ spores, showing conversion to + .

\section{Main data sources}

The best data on these evolution-related parameters come from fungi with tetrads or octads, where all products of a meiosis can be scored for segregation ratios, including conversion to wild-type and to mutant. Where a post-meiotic mitosis gives eight products in an octad, post-meiotic segregation is easily detected; additional testing can detect post-meiotic segregation in tetrads, as in yeast data of Fogel et al. (1979). In Drosophila and Zea, one can estimate $c$ but not $b$ or $d$.

Within data sets, mutations with inadequate sample sizes were omitted. Where many studies have been made on a locus because it generally had high $c$ values, as with the $b 2$ locus in Ascobolus immersus, later data sets were generally omitted to minimise biasing the summed data for an organism. In some fungal sets, the $0: 8$ class was omitted where there was evidence of bias (e.g., from phenocopies): omitting a few true $0: 8 \mathrm{~s}$ from this generally rare class makes little difference to the overall results. $8: 0$ s were also omitted in the analysis of yeast data from Fogel et al. (1979), in case of bias.

The following fungal data sources were used. Ascobolus immersus, European strains: loci $b 1$ and $b 2$, Leblon (1972); locus $Y$, Kruszewska and Gajewski (1967); locus 19, Mousseau (1966); locus 46, Gajewski et al. (1968); locus 75, Rossignol (1969); locus 84W, Paszewski and Prazmo (1969); 
locus 164, Baranowska (1970). Ascobolus immersus, Pasadena strains: Lamb and Ghikas (1979); Lamb and Helmi (1978); Howell (1982); Yu-Sun (1966), and unpublished data of Helmi, Hiremath, Lamb and Wickramaratne. Saccharomyces cerevisiae: Fogel, Hurst and Mortimer (1971); Fogel et al. (1979); Lawrence et al. (1975); Fink and Styles (1974). Schizosaccharomyces pombe: Gutz (1971). Sordaria brevicollis: buff (i), Ahmad (1975), $25^{\circ} \mathrm{C}$ data only; buff (ii) and (iii), MacDonald and Whitehouse (1979), crosses at $25^{\circ}$; set (iii) is identical with (ii) but omits the atypical YS 17 result; buff (iv), Sang and Whitehouse (1979), 30 . Sets (i), (ii), (iii) and (iv) are for the same locus, from the same laboratory, and have some mutations in common, though often with different results. Sordaria fimicola: $g$ locus, Kitani and Olive (1967); Whitehouse (1974); $i$ locus, Kitani (1982). References for minor fungal data sources e.g., for Neurospora, Podospora and Venturia, are given in the text, as are those for Drosophila melanogaster, D. virilis and Zea mays. Other sources were reviewed by Catcheside (1977) and Whitehouse (1982). Some data not included in the main survey are mentioned where they provide useful additional information, especially those of Rossignol, Paquette and Nicolas (1979).

\section{Distributions AND Limits for $c$ VAlUes FROM FUNGi}

As shown in table $1, c$ values had enormous variation for different mutations, within and between organisms, with limits from 0.0004 to 0.284 (i.e., gene conversion frequencies of 0.04 per cent to 28.4 per cent). Low frequencies of phenocopies, new mutations and reversions make the lower $c$ values less reliable than the higher ones; very extensive tests for such biases were made in some studies, and corrected values are given here when available, e.g., for Baranowska (1970).

\section{(i) Single mutations under different conditions}

In table 1, the Ascobolus immersus data from Pasadena strains for two locus $w$ I mutations, base-substitution $w \mathrm{I}-78$ and frame-shift $w \mathrm{I}-3 \mathrm{Cl}$, show $c$ values with 30 -fold and 18 -fold variation for the same mutation under different conditions. Different genotypes for conversion control factors ( $c c f s$ 2,3 and 4 ) caused $w I-78$ to have a range of conversion frequencies from 0.9 per cent to 26.5 per cent (Lamb and Helmi, 1978; Helmi and Lamb, 1981, 1983). In the European strains of $A$. immersus, Girard and Rossignol (1974) found $c v$ factors changing conversion frequencies up to several hundred-fold. In both Ascobolus strains, the different alleles of the control factors were probably present in the original strains isolated from the wild, so $c$ for a site may vary between individuals in a natural population.

Environmental factors can also affect $c$ : up to 4-fold changes occurred over a range of temperatures for buff mutations in Sordaria brevicollis (Ahmad, 1975), with even larger changes for $g$ and $h$ in S. fimicola (Lamb, 1969) and $w I-78$ and $w I-10$ in A. immersus (Wickramaratne and Lamb, 1978). The stability of conversion properties with time was considered by Lamb and Helmi (1982). As $c$ can vary with environment and background genotype, comparisons between different mutations and different loci should be made for comparable conditions. 


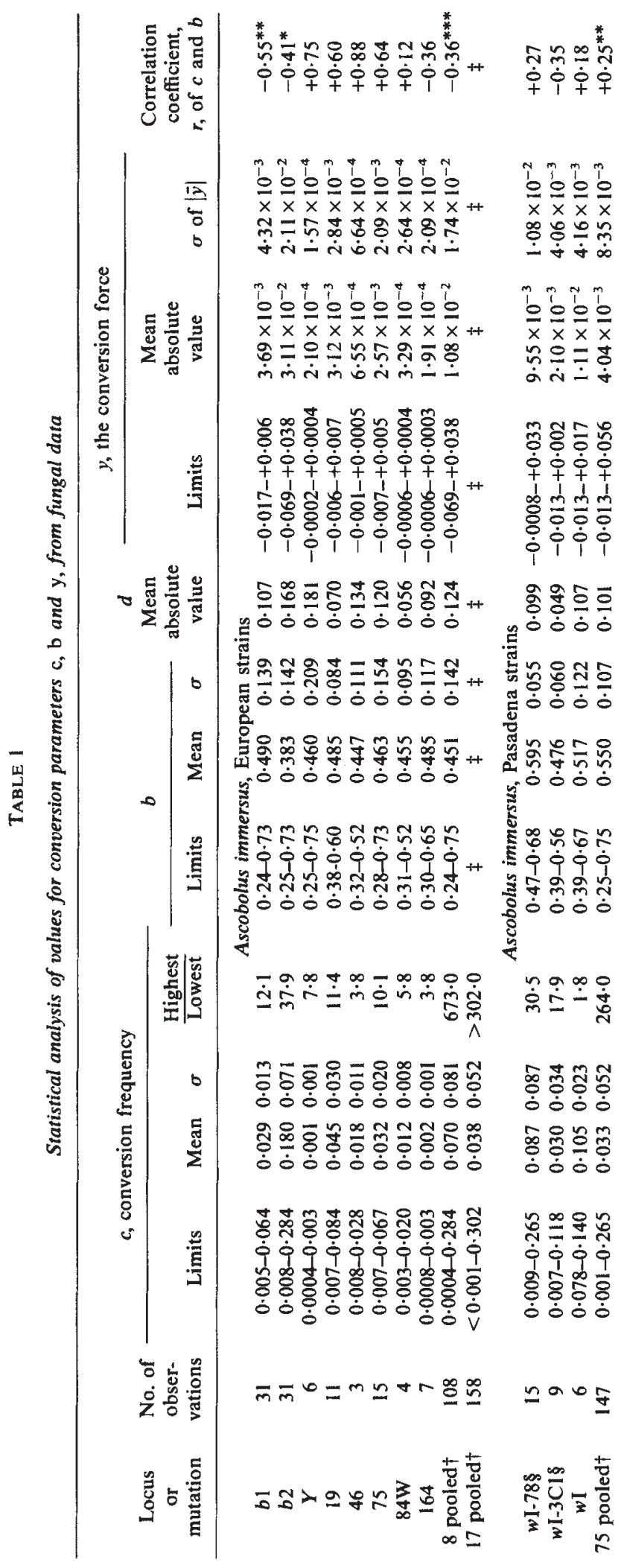




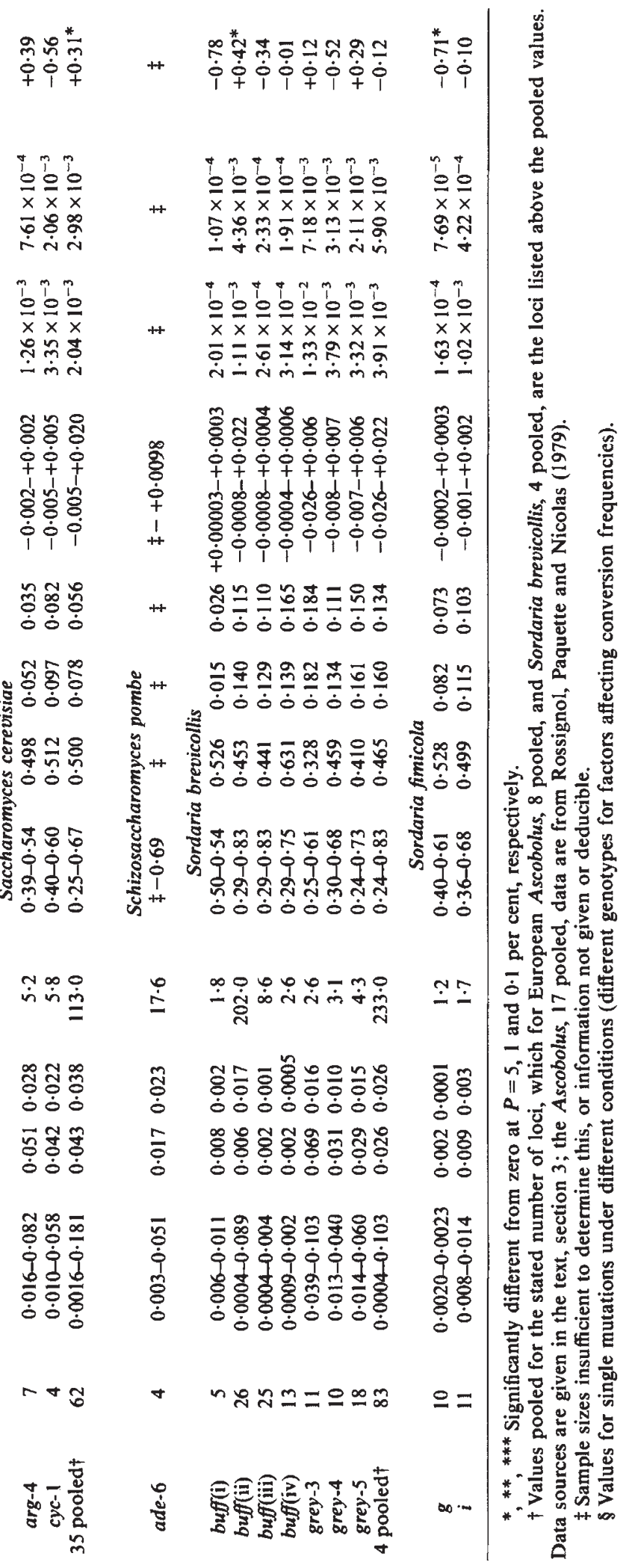


(ii) Different mutations at the same locus-tetrad and octad data

Data from fungi are given in table 1. The variation between different mutations at a locus ranged from almost none for $g$ in Sordaria fimicola, with only a $1 \cdot 2$-fold variation between highest and lowest values, to a great deal, as for $b 2$ in $A$. immersus with a 38 -fold difference. Most loci showed 1.5 to 15 -fold variation for $c$. One mutation with an extreme $c$ can make an enormous difference: for buff in Sordaria brevicollis, variation changed from 8.6-fold for 25 observations in (iii), to 202-fold in (ii), when YS 17 is included. In the Pasadena $\boldsymbol{A}$. immersus data, variation for six mutations at the $w I$ locus, obtained under similar conditions of temperature and genotype $\left(17.5^{\circ}, c c f-2(\mathrm{P})\right.$, non-super) was less than that for individual mutations $w \mathrm{I}-78$ and $w \mathrm{I}-3 \mathrm{Cl}$ under different genotypic conditions.

Different mutations at a locus, under the same conditions, can have very different $c$ frequencies. Table 2 shows Ascobolus and Sordaria data for presumed frame-shifts and presumed base-substitutions, to see if the molecular nature of a mutation affected its conversion properties. For three of the five loci, there were no significant differences in mean $c$ for these types of mutation. For grey-5 in $S$. brevicollis, the base-substitutions had a significantly higher mean $c$ than the frame-shifts, but the reverse situation occurred for $b l$ in $A$. immersus. The molecular nature of the mutations can therefore affect $c$ but more data are required to establish whether one type generally has higher $c$ values than the other.

The statistical distributions of $c$ for different mutations at a locus are shown in table 3 , displaying the data numerically rather than graphically because of problems of scale. 24 equal intervals were used, from just below the lowest $c$ to just above the highest $c$ at that locus. In general, mutations at a single locus showed a fairly even spread of values, with no tendency to the central clustering that would occur in a "normal distribution". The spread was roughly symmetrical for most loci, e.g., buff (iii) in S. brevicollis, though others were skewed, e.g., with more values near the higher limit for $b 2$ in $A$. immersus. One extreme value can change a distribution radically, as for buff in S. brevicollis where the inclusion of YS 17 in buff (ii) but not buff (iii) changes the upper limit, the interval size and the whole shape of the distribution. The buff (ii) set shows that $c$ distributions can be discontinuous, though most distributions might be continuous if samples were large enough. Different conversion control factor genotypes can cause discontinuous distributions, as for $w$ I-78 and probably the whole $w$ I locus in $\boldsymbol{A}$. immersus. Data on conversion frequency distributions in 17 loci in $\boldsymbol{A}$. immersus displayed in fig. 10 of Rossignol, Paquette and Nicolas (1979) showed similar effects to those in table 3 here.

\section{(iii) Different mutations at the same locus: single-strand and half-tetrad analyses}

$c$ can only be obtained from tetrad and octad data: it cannot be obtained directly from studies of single meiotic products. Single-strand analysis for gene conversion has been used in Drosophila, Zea and Neurospora (see Catcheside, 1977; Whitehouse, 1982), and half-tetrad analysis has been used in Drosophila (Smith, Finnerty and Chovnick, 1970). Selective methods were used to detect wild-type recombinants $\left(a^{1+} a^{2+}\right)$ from repulsion-phase heteroallelic crosses $\left(a^{1+} a^{2-} / a^{1-} a^{2+}\right)$, usually with flanking markers to 


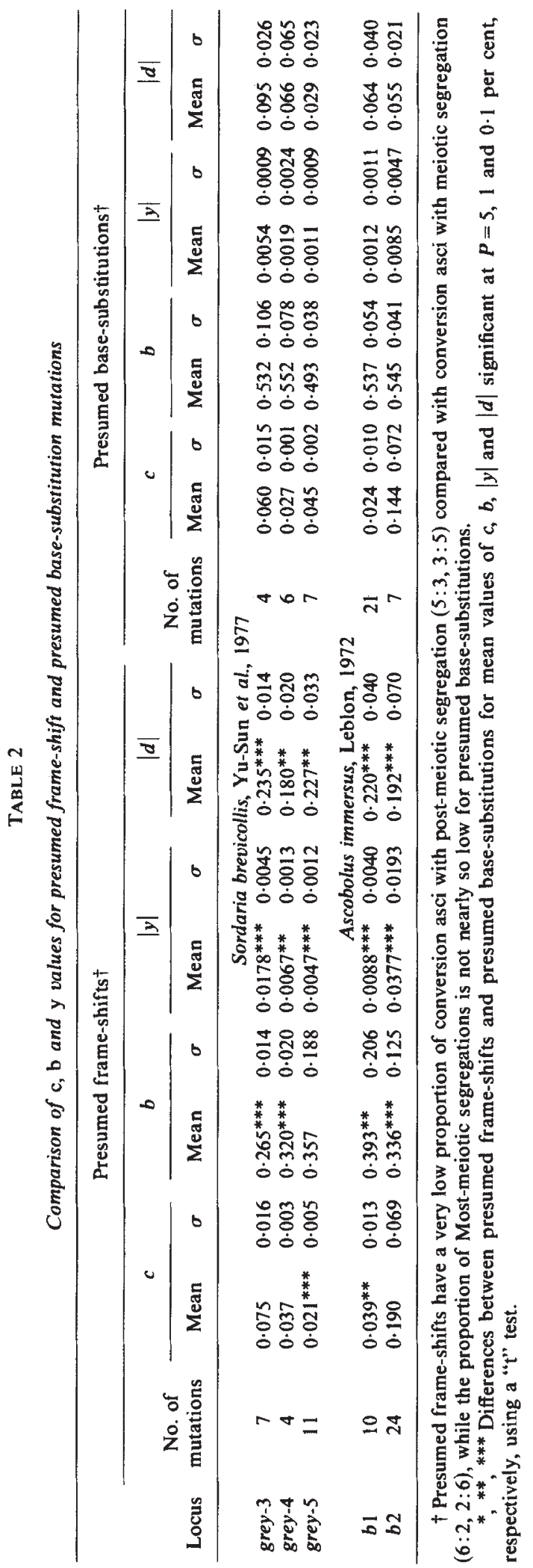




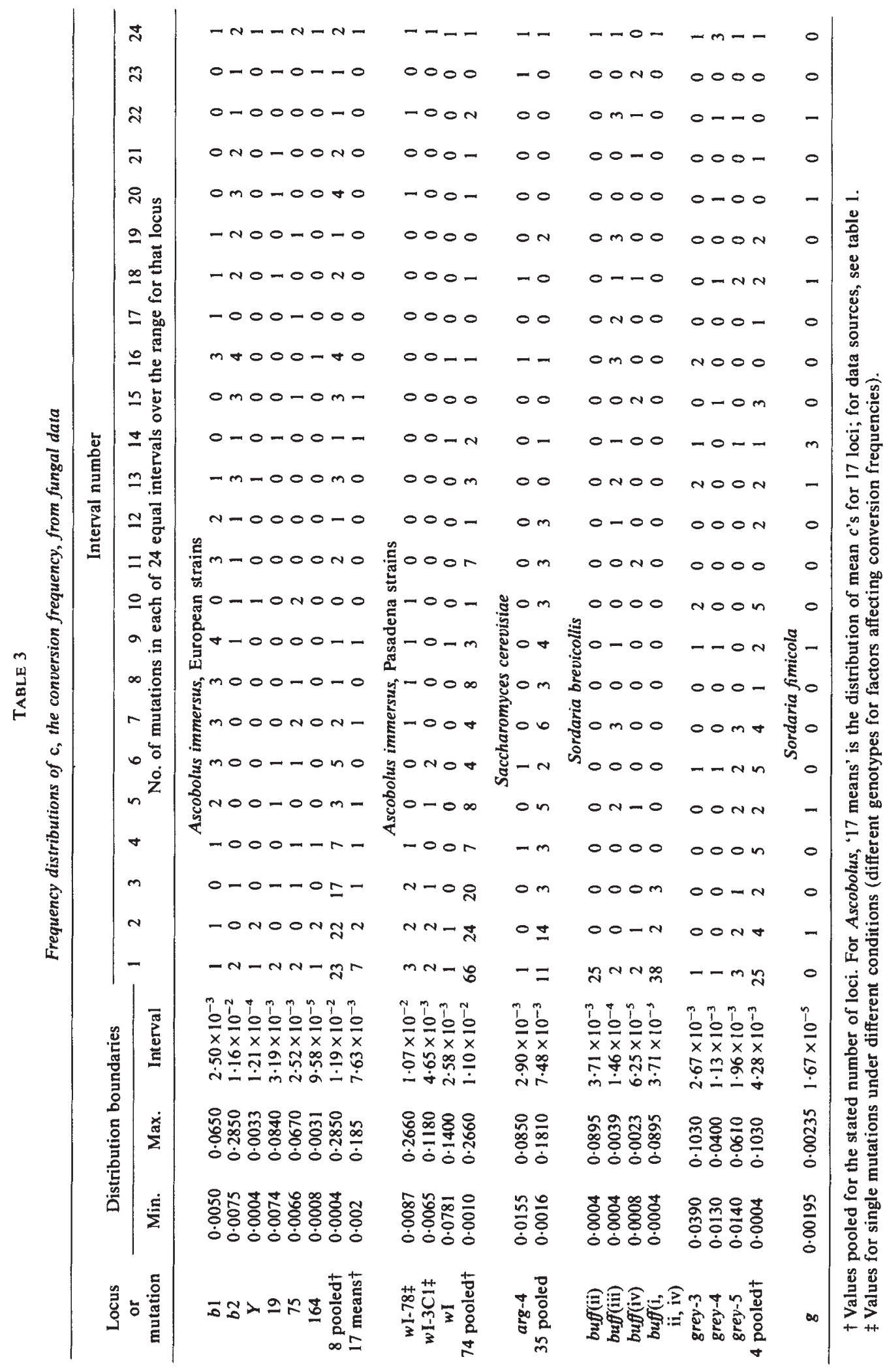


distinguish between wild-types produced by conversion to wild-type at $a^{1}$ or at $a^{2}$, and wild-types produced by a reciprocal crossover between $a^{1}$ and $a^{2}$. The term "conversion frequency" has been used by Drosophila workers for the frequency of conversion-produced wild-type progeny, but since that definition is quite different from that for $c$, the symbol $r c w$ will be used here for it, standing for "recombinants from conversion to wild-type".

$r c w$ and $c$ values are not directly comparable, for five reasons, most of them leading to $r c w$ being lower than $c$. Co-conversion at two heteroallelic sites would not give wild-type recombinants, though it could be detected in suitable tetrad analysis. In tetrad analysis, the whole tetrad or octad with an aberrant segregation ratio is scored as a convertant, while in single-strand and half-tetrad analysis only the phenotypically wild-type products (one from a $5: 3$ allele segregation at the converting site, two from a $6: 2$ segregation at the converting site) are scored as convertant. Single-strand and half-tetrad analyses normally omit all conversions to mutant as they do not give the selected wild-types, therefore on average missing half the total conversions ( $c$ includes conversions to mutant and to wild-type). Singlestrand analysis will also miss a significant fraction of conversions to wildtype where they are accompanied by nearby associated crossovers ( $25 \mathrm{per}$ cent to 75 per cent of conversions have associated crossovers-Fogel et al., 1979; Whitehouse, 1982) because the wild-type progeny may then be scored as coming from crossing-over, not conversion. These four considerations lead to $r c w$ values being on average about an order of magnitude lower than $c$ values (see (iv) below), and will be lower still if co-conversion is frequent.

The fifth consideration, differences in amount and direction of disparity in conversion direction, makes single-strand and half-tetrad analysis $r \mathrm{cw}$ values a very poor guide to different frequencies of meiotic conversion events at different sites within a locus. As shown here and by Lamb and Helmi (1982) disparities vary from nearly all conversion to wild-type, to nearly all conversion to mutant, for different mutations at a locus, yet only conversions to wild-type are scored in $r c w$ values. At the grey-5 locus in $S$. brevicollis (Yu-Sun et al., 1977) YS 25 and YS 16 both had $c=0.024$ but YS 25 had nearly all conversions to wild-type, giving an estimated $r c w$ equivalent of 0.0063 , and YS 16 had all conversions to mutant, so in selective single-strand analysis it would appear to show no detected conversions, with $r c w$ of zero. This shows how poor a guide $r c w$ values can be to true frequencies of conversion and hybrid-DNA (h-DNA) formation for different mutations at one locus.

At the rosy locus of Drosophila melanogaster, Hilliker and Chovnick (1981) reported significant differences in conversion frequency ( $r c w$, not $c$ ) for different mutations, ranging from $0.36 \times 10^{-6}$ to $22.4 \times 10^{-6}$ : a statistical analysis will not be given here as much of these differences could arise from different disparities for different mutations, as well as from different frequencies of h-DNA. The same criticism applies to other single-strand analysis data. The Drosophila half-tetrad studies, e.g., Smith, Finnerty and Chovnick (1970), are exactly like single-strand studies in using selective methods which only detect conversion to wild-type, so conversions to mutant are missed; conversion frequencies are scored as $r c w$, not $c$, and co-conversions are not detected. The same correction factor needed to estimate $c$ from $r c w s$ from single-strand analysis is therefore required for half-tetrad analysis, and 
indeed may also be required for any whole tetrad analyses which score numbers of wild-type recombinants by the same kind of selective technique.

(iv) Formulae to compare conversion frequency c from tetrad analysis with conversion frequency rcw from single-strand or half-tetrad analysis

One can estimate an equivalent $r c w$ value from tetrad or octad data which give $c$, but can not do the reverse to obtain $c$ from single-strand analysis data because of the missing information about conversion to mutant. Consider conversion at site $a^{1}$ only, in a repulsion-phase heteroallelic meiosis, $a^{1+} a^{2-} / a^{1-} a^{2+}$, giving octads. Where h-DNA forms in one chromatid only of a pair at this site, conversion ratios at this site $\left(a^{\prime}\right)$ of $8+: 0 m, 7+: 1 m, 6+: 2 m$ and $5+: 3 m$ will respectively give $4,3,2$ and 1 wild-type recombinant $\left(a^{1+} a^{2+}\right)$ spores in the octad. Therefore, for single chromatid (asymmetric) h-DNA,

$$
r c w=\frac{(8: 0 \times 4)+(7: 1 \times 3)+(6: 2 \times 2)+(5: 3 \times 1)}{\text { Total number of octads } \times 8} .
$$

The situation is more complicated when h-DNA forms symmetrically, in two chromatids of a pair, at the site. The 8:0,7:1,6:2 and 5:3 classes are as for the previous case, but 1 wild-type recombinant per octad will come from aberrant $4: 4$ octads and from $3: 5$ segregations with corrections by substitution, and 2 wild-type recombinants per octad will come from correction $4: 4 \mathrm{~s}$ where correction occurs by substitution (i.e., the wild-type base pair and the mutant base pair which became involved in h-DNA were respectively changed by correction to mutant and to wild-type), not by restitution (in which the wild-type base pair and mutant base pair involved in h-DNA are respectively corrected back to wild-type and to mutant). The formula for calculating rcw from octad data for symmetric h-DNA is thus as above but with the addition to the numerator of "substitution $3: 5+$ aberrant $4: 4+$ (substitution correction $4: 4 \times 2$ )". As some or all of the last three terms in the numerator are difficult to determine in most systems, the formula used will be that for single hybrid chromatid h-DNA; this is accurate for single hybrid chromatid h-DNA but will slightly underestimate $\mathrm{rcw}$ because symmetric (dual hybrid chromatid) h-DNA does occur and the three non-determined classes in the numerator can not be assumed to have zero values. The formula used may often overestimate $r c w$, however, as it does not allow for reductions in rcw from co-conversion.

The formula for $r w$ given above for single chromatid h-DNA has here been used on sets of fungal data, so that the observed $c$ values can be directly compared with the estimated $r c w$ values which would have been obtained if single-strand analysis had been done in the same crosses. The results are given in table 4 for a number of mutations at each locus. By comparing the mean values of $c$ with the mean values of $r c w$ at each locus, and from the mean value of $c$ divided by the mean value of $r c w$ (right-most column, table 4 ), it is clear that $r c w$ values consistently underestimate conversion frequencies relative to $c$ values, by factors averaging from 5.6 to 23.5 for the locus averages quoted, with an average factor of 11.6 for the nine loci. For individual mutations, the underestimation factor has a much wider range, from 3.8 to infinity. The effect of disparity in raising 


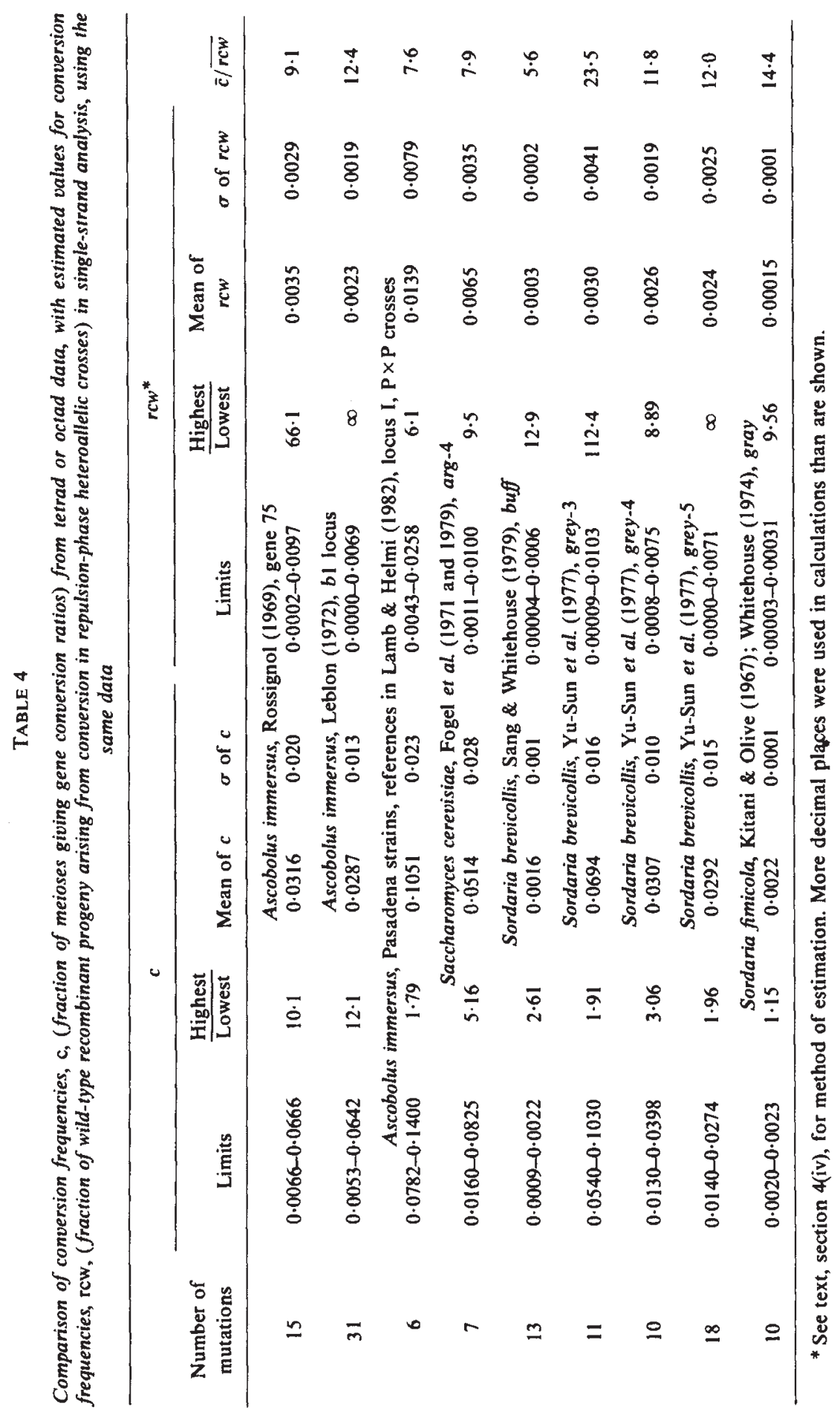


variability for rcws for different mutations at a locus, compared with their variability for $c$, is very clearly shown in the "highest/lowest" columns of table 4.

\section{(v) Mutations in an organism, pooling data from various loci}

The limits and means of pooled $c$ values can be compared in table 1 for different fungi: it is best to exclude $S$. pombe and $S$. fimicola as only one or two loci are given, which might not be typical. The minimum, mean, maximum and highest/lowest $c$ differ only a few fold between species, with much less difference between species than for different loci within a species. Mean $c$ varied from 0.026 in Sordaria brevicollis to 0.070 in European A. immersus.

Less extensive data are available from other species. In Neurospora crassa data summarised by Catcheside (1977), conversion events per locus had frequencies of 0.007 for $p d x, 0.01$ for pan-2, 0.017 for $c y s$, with $c$ for individual sites, in these multipoint crosses, of about a half or a third of those values, e.g., $c$ of $0.0068,0.0062,0.0056,0.0034$ and 0.0034 for pan-2 mutations. Catcheside (1977) gave other conversion data for Neurospora loci, giving these $c$ estimates: am-l, 0.009; cys, 0.012; his-1, 0.004; his-2, 0.008 ; his-3, 0.02; his-5, 0.0007; me-2, 0.008; pan-2, 0.006, which averages 0.0093 for these loci. In Venturia inequalis (Boone and Keitt, 1956), $c$ for N-46 was 0.146 . In Podospora anserina, $+\times m$ crosses canot be scored directly for $c$ as the four ascospores each contain two different nuclei, so one colour ratio can have several origins. Touré (1972) made two-point repulsion crosses involving 33 mutations of spore colour locus 14, where only conversions to wild-type were detected, and undetected co-conversions reduced observed conversion. The largest recombination frequencies for given sites were when those sites were crossed with more distant ones and were less likely to be so biased by undetected co-conversion: frequencies averaged 0.011 per cent for $A \times C$ crosses (Touré, 1972, table 1) and ranged from 0.003 per cent to 0.02 per cent for non-co-converting mutants in other crosses (his table 2). Because of unknown values of $b$ and $d$, one cannot express these directly in terms of $c$, but if one assumes that on average, half the conversions would have been to $m$, then $c$ of around 0.0002 seems likely for this locus, which is lower than for fungi previously quoted.

The Drosophila and Zea data consist of $r c w$ values (see section 4(iii)), and one can not reliably use a correction factor to change individual $r c w$ values into $c$, but as described in section 4(iv), one can use a multiplier of about 11.6 on mean $r c w$ values to get a mean $c$, although this still underestimates $c$ because of undetected co-conversion. This multiplier gives estimated mean $c$ values in Drosophila melanogaster of 0.000058 for rosy (data of Hilliker and Chovnick, 1981), 0.000034 for maroon-like (data of Smith, Finnerty and Chovnick, 1970), 0.00036 for rudimentary (data of Carlson, 1971, combining information in his fig. 2 and Table 7), 0.00031 for garnet (Hexter, 1958, quoted by Whitehouse, 1982) and 0.000068 for white (Green, 1960 , quoted by Whitehouse, 1982). In Drosophila virilis (data of Demerec, 1926 , quoted by Whitehouse, 1982) for the reddish locus, estimated $c$ is about 0.0183 in one experiment, more in another. $c$ values were therefore lower than in fungi for $D$. melanogaster, but $c$ was high in $D$. virilis: data on more loci and on a wider range of organisms are clearly desirable. 
An anonymous referee has pointed out that a rough upper limit on conversion frequency in Drosophila could be obtained from the frequency of "double-crossing over" in three-point test crosses with linked markers. Conversion at the centre locus alone would give a genotype as from a double crossover, with one crossover in each interval. This would actually miss all conversions accompanied by a crossover in the same strand, as the product would then resemble a single crossover type. Suitably large numbers of progeny were recorded in the results of Bridges and Olbrycht (1926), with double crossover progeny having frequencies of 0.02 per cent for $s c-6 \cdot 74-e c-9 \cdot 64-c v$ (the figures are observed recombination frequencies, per cent) and 0.5 per cent for $c t-16 \cdot 0-v-11 \cdot 1-g$, where the larger figure in the second cross probably represents more genuine double-crossover events rather than more conversions. To get $c$ equivalents, one would have to correct for scoring single strands, not tetrads, but in the above data conversions to mutant would have been detected, as well as to wild-type. For very close sites, co-conversion could affect the results significantly.

Intragenic recombination at the $A d h$ locus in Zea mays was studied by Freeling (1978), but without outside markers it was not possible to distinguish wild-types arising from conversion from those arising by reciprocal crossing-over. The values therefore give upper limits for conversion frequencies (as $r c w$ ) but actual values would be lower if recombinants also arose by crossing-over within the gene, although co-conversion could lead to underestimations. Using the multiplier discussed above, upper limits of estimated $c$ are about 0.00097 for $A d h 1-S$ alleles and about 0.00011 for Adhl-F alleles. The waxy locus studies of Nelson (1962) on Zea did include outside markers: from $W x$ kernels with parental outside marker arrangements, one can calculate $c$ estimates of 0.00285 and 0.00159 . Subsequent work (Nelson, 1975) suggests these are underestimates (Whitehouse, 1982, p. 254). The $Z e a$ values are thus comparable to the lower values shown for fungi in table 1, but are higher than those for Drosophila melanogaster.

\section{Distributions AND Limits for $b$ AND $d$ VAlues}

$b$ and $d$ cannot be estimated from single-strand analysis, so the relevant data are all from fungal octads or tetrads. Because disparity, $d$, is so directly related to $b, b$ and $d$ will be analysed together.

\section{(i) Single mutations under different conditions}

The $\boldsymbol{A}$. immersus data in table 1 show $\boldsymbol{b}$ ranging from 0.47 to 0.68 for base-substitution $w \mathrm{I}-78$ and from 0.39 to 0.56 for frame-shift $w \mathrm{I}-3 \mathrm{Cl}$, under different genotypic conditions. The direction and extent of disparity can therefore be changed by background genotype, and temperature can also reverse the direction of disparity, as shown by Wickramaratne and Lamb (1978) for $w \mathrm{I}-10$ in $\boldsymbol{A}$. immersus. In the same organism, very large changes in extent and direction of disparity in crosses with different conversion control factors were shown by Lamb and Ghikas (1979) for NGw5, BBw21, BHo and BB9w8. Clearly $b$ and $d$ are not fixed properties of a mutation, and comparisons of different mutations at a locus should be made under similar conditions of environment and genotype. 


\section{(ii) Different mutations at the same locus}

From the limits of $b$ for single loci in table 1 , it is clear that different mutations at a single locus can have extremely different $b$ and $d$ values. As discussed by Lamb and Helmi (1982), the theoretically expected extreme upper and lower values of $b$ are about 0.75 and $0.25(d=+0.25$ and -0.25 , respectively), so mutations at the same locus were fairly often near the opposite ends of possible $b$ limits, e.g., A. immersus loci $b 1, b 2, Y, 75, S$. brevicollis loci buff, grey-5, in table 1 . All loci in table 1 have some mutations with $b>0.5$ (disparity favours wild-type) and some with $b<0.5$ (disparity favours mutant). $b$ values are clearly a property of individual mutations (more accurately, of a particular mutation and a particular wild-type allele, or a pair of alleles), not of loci as a whole $b$ values from fungi not in table 1 were similar: Venturia inequalis, N-46, $b$ is 0.35 ; Neurospora crassa, 5 alleles of pan-2 (data quoted by Catcheside, 1977) had $b$ of $0.77,0.44,0.63$, 0.43 and 0.50 , though sample sizes were small. In Schizosaccharomyces pombe, only ade-6-M26 had a large enough sample, with $b$ of 0.692 , with clearly significant positive disparity (46 3:1 tetrads, $61: 3$ tetrads).

The distributions of $b$ for different mutations at a locus are shown in table 5. Unlike table 3 for $c$ distributions, the same minimum $(0 \cdot 220)$ and maximum $(0 \cdot 840) b$ could be used for all loci, giving more direct comparability between different results. The distributions for single loci are much too flat and dispersed to fit "normal distributions", e.g., A. immersus, locus 164, $S$. fimicola, $g$ locus. Although larger samples would be helpful, some loci appear to have bi-modal ( $A$. immersus, $b$ l) or multi-modal ( $S$. brevicollis, buff) distributions, and/or modes not central (S. brevicollis, grey-5) and/or discontinuous distributions (S. brevicollis, grey-3).

The Ascobolus and Sordaria data in table 2 show highly significant effects of molecular type on $b$ values, for four out of five loci. For all five loci, mean $b$ was much lower for frame-shifts $(0.265$ to 0.393$)$ than for base-substitutions $(0.493$ to 0.552$)$. For all five loci, the mean absolute disparity was significantly higher for frame-shifts $(0 \cdot 180-0 \cdot 235)$ than for base-substitutions $(0.029-0.095)$, at $P=1$ per cent. The cluster of values at the low end of the $b$ range in table 4 is largely, though not entirely, due to frame-shifts, while the cluster around central values of $b$ is largely, though not entirely, due to base-substitutions. The very marked disparity in favour of conversion to mutant $(b<0.5)$ in both Ascobolus and Sordaria data in table 2 for frame-shifts raises the question as to whether frame-shifts all have preferential conversion to mutant. This is not the case: in $A$. immersus at the $b l$ locus, three out of 10 frame-shifts have $b>0.5$, namely $0.68,0.65$ and 0.73 , and at the $b 2$ locus, two out of 24 frame-shifts have $b$ of 0.73 and 0.62 . In $S$. brevicollis, all seven frame-shifts at grey-3 have low $b$, $0 \cdot 25-0.29$; so do the four at grey-4, 0.30-0.34, while at grey-5, two out of 11 frame-shifts have high $b, 0.73$ and 0.73 . Unfortunately it is unknown whether all frame-shifts with low $b$ values were of the same sign (e.g., all single base-pair additions), with those of high $b$ being of opposite sign (e.g., all single base-pair deletions). A case of two frame-shifts of opposite sign is included in the Pasadena $A$. immersus data, where $w \mathrm{I}-\mathrm{wSu}^{+}$, which has $b$ of 0.67 , is an intragenic suppressor of frame-shift $w \mathrm{I}-3 \mathrm{Cl}$, which has $b$ of 0.39 ; these two frame-shifts of opposite sign thus have opposite directions of disparity. 


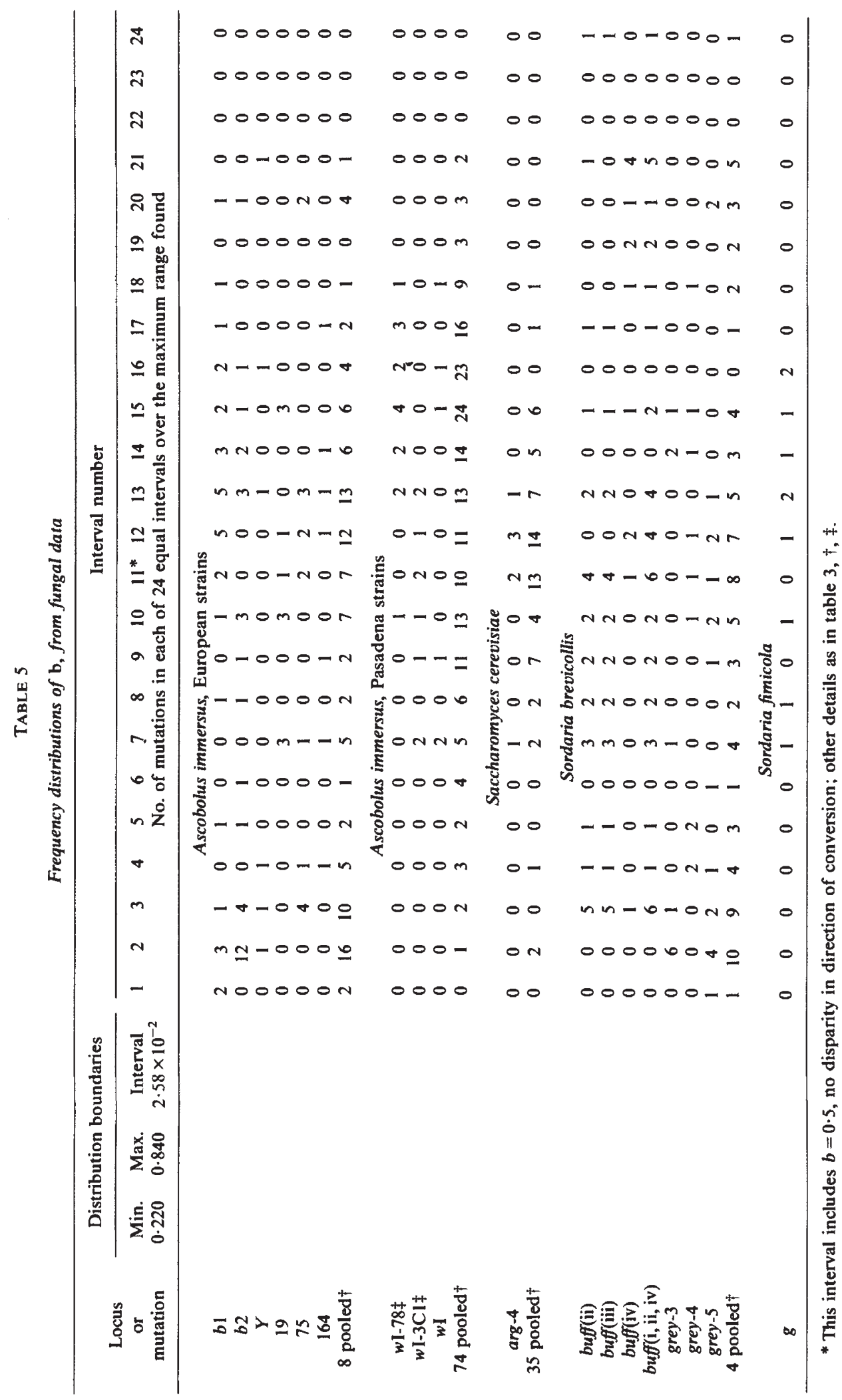


Excellent data on frame-shift disparity were given by Rossignol, Paquette and Nicolas (1979) for $b 2$ in $A$. immersus: the measure $6+: 2 m / 2: 6$ in their fig. 8 can be changed to $b$ by halving it and adding 0.25 , as no or very few octads with post-meiotic segregation occurred. Frame-shift El, $b$ of about 0.28 , had two intragenic suppressors with $b$ of about $0.7 ; A O, b$ of about 0.28 , had four intragenic suppressors with $b$ of 0.65 to $0.73 ; \mathrm{FO}, b$ of about 0.4 , had 13 intragenic suppressors, $b$ of about 0.55 to 0.63 . In all these cases the suppressors must be of opposite sign to the original frameshift. These results are consistent with frame-shifts of opposite sign always having opposite directions of disparity, though it is unknown which were additions and which were deletions. The extent of the disparity for frameshifts seemed correlated with position of the site within the $b 2$ locus, which can be related to different frequencies of asymmetric and symmetric h-DNA at different positions: see Rossignol, Paquette and Nicolas (1979) for discussion. Their data also showed lower disparities for presumed base-substitutions than for frame-shifts.

The Saccharomyces cerevisiae data of Fogel et al. (1979), Fink and Styles (1974) and Lawrence et al. (1975) enable one to compare the properties of large deletions with those of point mutations at the same loci. The first two sets of data are on the his-4 locus: of two point mutations, one has significant $(P<1$ per cent) disparity favouring wild-type, $d=+0.034$, the other has disparity favouring mutant, $d=-0.058$, but not significant at $P=5$ per cent. The deletions are mainly 300-400 nucleotides long: two data have significant disparity favouring wild-type, $d=+0.112(P<1$ per cent), and +0.153 $(P=2-5$ per cent), and three data have disparity favouring mutant, $d=$ $-0.066(P=5-10$ per cent $),-0.079(P=5-10$ per cent $)$ and -0.063 ( $P$ not significant), where the lack of significance for these three sets is related to sample size, which is only 8 to 49 conversion asci. In the data of Lawrence et al. (1975) on the cyc 1 locus, cyc 1-1 has deletion of the whole locus; two asci showed conversion to wild-type, one showed conversion to mutant, so the sample is too small to assess disparity. For these three sets of data, large deletions have a mean absolute $d$ of $0.093(\sigma 0.034)$ compared with $0.048(\sigma 0.052)$ for the remaining yeast mutations in table 1 , nearly all of which are probably point mutations. Large deletions are thus capable of showing significant disparity, may have disparity favouring wild-type or mutant, and in the small sample of results available, generally had higher absolute disparities than point mutations. Like frame-shifts which also give non-pairs in h-DNA, the two large deletions in Fogel et al. (1979) data gave very few (actually none) post-meiotic segregations.

\section{(iii) Mutations in an organism, pooling data from various loci}

The limits of $b$ in pooled data in table 1 were generally similar in different organisms, ranging from near the lowest values expected to near the highest values expected, $0.25-0.75$, occasionally exceeding that range but with a lower top value, 0.67, for Saccharomyces. The pooled data from Pasadena strains of $A$. immersus gave a roughly unimodal distribution of $b$ in table 5 , with more values above 0.5 than below it, and a mean of 0.550 (table 1 ). By contrast, the European $\boldsymbol{A}$. immersus distribution had at least two clear modes, one near 0.5 and one near 0.26 , with a mean of 0.451 . The pooled $S$. brevicollis data distribution is tri-modal, with modes at about $0.26,0.5$ 
and $0 \cdot 75$. Values of $b$ (and $d$ ) towards either extreme can thus be very common. These differences between data sets, and the apparent differences in mean $b$ for different loci within an organism (table 1), need careful interpretation because clear differences were shown in section 5(ii) between frame-shifts and base-substitutions, which are represented to different extents at different loci and in different data sets. For example, in European $A$. immersus, mutations at loci $Y, 19,46,75,84 \mathrm{~W}$ and 164 were probably all spontaneous; mutations at $b l$ were 5 spontaneous, 5 (induced by) ICR-170, 10 NMG, 11 EMS, with 10 presumed frame-shifts and 21 presumed base-substitutions, while corresponding numbers for $b 2$ were $5,18,3$ and 5 , with 24 frame-shifts, 7 base-substitutions. Of 10 spontaneous mutations at $b 1$ and $b 2$, six are presumed frame-shifts. It is the $b 2$ locus, with the high proportion of frame-shifts, which has the lowest mean $b$ in Ascobolus. This low mean $b$ could be explained if most of the frame-shifts were of the same sign (e.g., if the mutagen produced mainly additions) and if ones of the same sign tend to the same end of the $b$ range.

The $b$ distributions for individual loci look much flatter (negative kurtosis) than a typical normal distribution, though the numbers of observations are too small for proper testing. On pooled data for an organism one can calculate moments of skewness and kurtosis from adequate sample sizes, for example, kurtosis coefficients of 1.9 and $2 \cdot 1$ respectively for pooled data from European $A$. immersus and $S$. brevicollis; both are less than the expected 3.0 for a normal distribution, but significance tests based on unimodal normal distributions are of doubtful validity when both these distributions appear bi-modal.

The best way to compare the extent of disparity in conversion direction is from mean absolute $d$ values in table 1 , not $b$. There were clear differences in mean absolute $d$ for loci within an organism, e.g., for grey-3 and grey-4 in $S$. brevicollis, and 19 and $b 2$ in European $A$. immersus. Care is needed in interpretation, as frame-shifts and base-substitutions differ in mean absolute $d$ (table 2), and are represented to very different extents at different loci and in data from different organisms. Mean absolute $d$ ranged from 0.056 to 0.134 in pooled data from different fungi (table 1), with an overall average of 0.114 . This is very high compared with a maximum expected disparity of 0.25 for any mutation, confirming how extensive disparity is in these fungi. The mean absolute $d$ for Saccharomyces was 0.056 , compared with values of 0.101 to 0.134 for Sordaria brevicollis and Ascobolus immersus. This difference can be largely or wholly explained by the yeast data containing no known frame-shift mutations (Fogel et al., 1979 , p. 1330), consisting largely of base-substitutions and a few large deletions. Table 2 shows that in Sordaria brevicollis and Ascobolus immersus mean absolute $d$ values averaged $0.029,0.055,0.064,0.066$ and 0.095 for various loci when only presumed base-substitutions are considered. These are comparable to the yeast value, while the presumed frame-shifts typically had much higher values.

\section{Possible CorRelations Between $b$ AND $c$}

Correlation tests were made on $b$ and $c$ as correlations (although not expected theoretically) would affect predictions of $y$ distributions for organisms where only $c$ can be estimated, not $b$. As shown in table $1, r$ was 
usually not significantly different from zero; significant positive and negative correlations occurred for some loci, though the significant values of $r$ were very variable and not near $1 \cdot 0$ or $-1 \cdot 0$. Such results are difficult to interpret: the deviations from normality for distributions of $b$ and $c$ (tables 5 and 3) reduce the accuracy of this test, which is based on assumptions of bivariate normality.

\section{Distributions and Limits for $y$ VAlues}

As $y=c(b-0 \cdot 5)$, and $b$ and $c$ were not generally correlated, the $y$ distributions are a combination of those for $c$ and $b$.

\section{(i) Single mutations under different conditions}

The $A$. immersus data in table 1 show $y$ ranging from positive to negative for both mutations $w \mathrm{I}-78$ and $w \mathrm{I}-3 \mathrm{Cl}$ under different genotypic conditions. Which allele of a pair is favoured by gene conversion, as well as the extent of favouring, can therefore change with background genotype, and might differ between individuals in a natural population. The previously discussed effects of temperature on $c$ and $b$ mean that $y$ for a given mutation depends on environment as well as genotype.

\section{(ii) Different mutations at the same locus}

As shown in table 1, all loci had some mutations with positive $y$ and some with negative $y$; most loci also had some mutations with $y$ of about zero, as expected from the distributions of $b$ in table 5 . Where there are large variations at a locus, e.g., from -0.069 to +0.038 for $b 2$ in A. immersus, some mutant alleles are strongly favoured-relative to wild-type-by conversion, while other mutant alleles are strongly disfavoured.

Table 6 shows the distributions of $y$ for mutations at particular loci. The general trend was a dispersed distribution, with no central concentration of values, unlike a "normal distribution", e.g., b2 in Ascobolus, arg-4 in yeast, grey-5 in $S$. brevicollis. The importance of this is that extreme and fairly extreme values of $y$, both positive and negative, where conversion has its greatest effect on allele frequencies, are quite common. For single loci, values are not clustered closely around zero.

From table 2, it is seen that there were highly significant differences in mean absolute values of $y$ for different types of mutation in Ascobolus and Sordaria, for all 5 loci tested. Such values were generally 3 to 8 times higher for frame-shifts than for base-substitutions, due largely to differences in mean disparities rather than to differences in $c$, so frame-shifts could be more affected by effects of conversion on allele frequencies than are basesubstitutions. This could be offset, however, by a reverse effect concerning selection coefficients: frame-shifts usually give complete lack of gene function, while base-substitutions can give cryptic mutations (Lamb, 1975) and tolerated amino acid changes, so frame-shifts could on average have higher selection coefficients against them than do base-substitutions.

\section{(iii) Mutations in an organism, pooling data from various loci}

$y$, like $b$, is largely a property of a pair of alleles, not of a locus as a whole, but because $c$ is partly a property of a locus, different loci often 


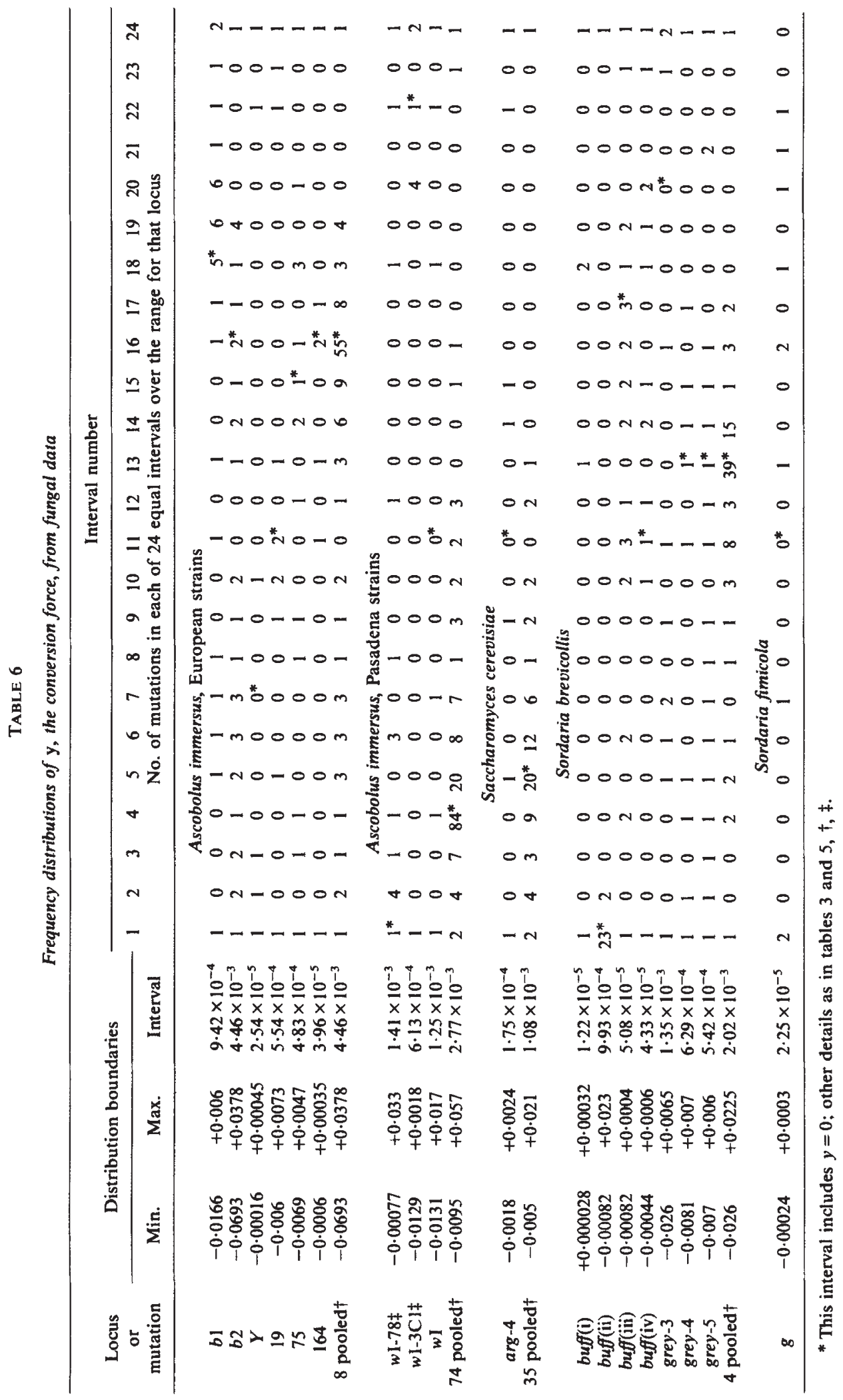


have different limits of $y$. Thus loci with a low mean $c$, such as $g$ in $S$. fimicola and $Y$ in $A$. immersus, tend to have low deviations from $y=0$, and loci with a high mean $c$, such as $b 2$ and $w I$ in $A$. immersus, tend to have high mean absolute values of $y$ and hence greater effects of conversion on allele frequencies. The mean absolute $y$ varied from $1.63 \times 10^{-4}$ for $g$ in $S$. fimicola to $3 \cdot 11 \times 10^{-2}$ for $b 2$ in $A$. immersus. The most extreme $y$ favouring conversion to mutant was -0.069 and the largest in favour of wild-type was +0.056 , both from $A$. immersus; Lamb and Helmi (1982) suggested that -0.1 and +0.1 were the most extreme $y$ values that might be encountered. For fungi not included in table $1, y$ values were similar to those in table 1 , e.g., $-2.19 \times 10^{-2}$ for N-46 in Venturia, $+1.83 \times 10^{-3},-3.53 \times 10^{-4},+8.59 \times$ $10^{-4},-2.58 \times 10^{-4}$, and 0 , for pan-2 alleles in Neurospora.

For pooled data in an organism, table 6, the distributions of $y$ showed much more clustering, usually with a sharp peak around $y=0$, than for mutations at a single locus, but with more values near the extremes than for a normal distribution. The European $A$. immersus and the Pasadena $\boldsymbol{A}$. immersus values are both skewed, but in opposite directions, largely because of their differently skewed $b$ values (table 5).

Because of the lack of information on $b$ from single-strand analysis, one cannot give $y$ values for Drosophila or Zea, nor from the tetrad data from Podospora. If the spread of $b$ values in those organisms were similar to that in Ascobolus, Sordaria and Saccharomyces, then their mean absolute $y$ values would be related to those in Table 1 in proportion to their $c$ values. Estimated mean absolute $y$ values would then range from 0.0000041 to 0.000041 for different loci in Drosophila melanogaster, 0.0021 for $D$. virilis, and 0.00001 to 0.00034 for Zea mays, compared with observed fungal values of 0.0108 (limits -0.069 to +0.038 ) for 108 mutations in European strains of Ascobolus immersus, $0.0040(-0.013$ to +0.056$)$ for 147 values in Pasadena strains of $A$. immersus, $0.0020(-0.005$ to +0.020$)$ for 62 values in Saccharomyces cerevisiae, $0.0039(-0.026$ to +0.022$)$ for 83 values in Sordaria brevicollis, $0.0006(-0.001$ to +0.002$)$ for 21 values (only two loci) in $S$. fimicola, and $0.0007(-0.0004$ to +0.0018$)$ for five pan-2 mutations in Neurospora crassa.

\section{Discussion}

Different mutations at a locus often have quite different conversion frequencies. The $c$ for a mutation will depend on the locus, on its position within the locus in relation to fixed or preferred sites of initiation of h-DNA (Rossignol, Paquette and Nicolas, 1979, and Whitehouse, 1982), and on the correction properties of the mispair (base-substitutions) or non-pair (e.g., frame-shifts) produced by a heterozygous mutation in h-DNA. The correction properties include the probability of correction and the direction of correction (to + or to $m$, or to $A$ or to $a$ ); these affect $c$ (and $b, d$ and $y$ ) by determining whether the h-DNA gives rise to a detectable conversion class such as $6: 2,2: 6,5: 3$ or $3: 5$, or "correction $4: 4 s$ " which are often not distinguishable from normal $4: 4 \mathrm{~s}$ (with no h-DNA formation at the site) or to the aberrant 4:4 class. A higher probability of correction could lower observed $c$ if it caused many potential conversion asci-especially $5: 3$ and $3: 5$-to give "correction $4: 4 \mathrm{~s}$ " from either symmetric or asymmetric 
h-DNA: for symmetric h-DNA, this is most likely for low disparity mutations as it then requires one correction to mutant, one to wild-type. It could increase $c$ if potential aberrant $4: 4 \mathrm{~s}$ (from symmetric $\mathrm{h}-\mathrm{DNA}$ ), in systems where they are not detected, were corrected to give detected conversion classes such as $6: 2$ or $3: 5$. An ability to detect aberrant $4: 4$ s would not change values for $y$ as allele ratios in such octads are the same as in normal $4: 4$ octads.

There were significant differences in mean $c$ between loci within an organism, presumably reflecting different mean frequencies of h-DNA in different regions of the genome. The Ascobolus data of Rossignol, Paquette and Nicolas (1979) show no consistent relation between centromere distance and conversion frequency. The existence of significant differences in mean $c$ between different organisms is more difficult to establish, partly because of the difficulty of comparing $c$ values from fungal octads and tetrads with rcw from single-strand analysis, and partly because of the low number of loci studied in most non-fungal organisms. In Drosophtla melanogaster data quoted in section 4(v), the estimated mean $c$ equivalents of 0.000034 to 0.00036 for five loci were lower than typical fungal values of 0.0004 to 0.302 (table 1), yet the one Drosophila virilis $c$ was about 0.0183 . More data on different Drosophila species and loci are clearly required before one can establish general trends, especially for species not having the suppression of crossing-over in the male that occurs in $D$. melanogaster and which might have some influence on recombination in females. In Zea, $c$ estimates were about 0.002 to 0.003 for $W x$, and under 0.001 and under 0.0001 for $A d h$ alleles. Even within the fungi, between-organism differences are hard to establish. For example, the $g$ locus $c$ values for Sordaria fimicola appear low for fungi, at about 0.002 (table 1), yet in that species a $g$ allele had $c$ ranging from 0.009 at $10^{\circ}$ to 0.106 at $27.5^{\circ}$, and the $h$ allele at the hyaline locus had $c$ of 0.035 to 0.133 at different temperatures (Lamb, 1969).

Different mutations at a locus often had quite different extents and directions of disparity, and frame-shifts tended to have higher disparities (section 5(ii)) and higher frequencies of correction than base-substitutions. Disparity values and $b$ values near both extremes (strongly preferential conversion to wild-type or to mutant) were common at most loci in Ascobolus and Sordaria brevicollis. Different mean $b$ and $d$ for different loci occurred and are probably related to different proportions of different types of mutant (especially frame-shifts versus base-substitutions) at the loci, as different loci had very different proportions of spontaneous mutations and of ones induced with different types of mutagen.

There is a common view, as in Fincham, Day and Radford (1979, p. $231)$, that yeast generally shows parity in direction of conversion $(b=0.5$, $d=0$ ) and that therefore mismatch recognition must be different in yeast compared with Ascobolus and Scordaria. One factor was the typically small sample sizes of conversion asci in the early yeast study of Fogel et al.(1971), as those mutations with only 2 to 10 conversion asci are unlikely to show statistically significant disparity. Thus pet 1 showed no disparity from 4 conversion asci in the early study, but in the larger study of Fogel et al. (1979) the $46: 2$ and $272: 6$ asci for pet 1 showed disparity significant at $P=1$ per cent. The larger study of yeast showed significant disparity at 12 out of 30 sites, and some non-significant values might well have been 
significant from larger samples (arg4-4, ade6). At the end of section 5(iii) here it was shown that the mean absolute disparity for yeast was comparable to mean absolute disparities for presumed base-substitutions from Ascobolus and Sordaria, and that the yeast data contained no known frame-shifts (Fogel et al., 1979, p. 1330), being mainly from base-substitutions and a few large deletions. Table 2 shows clearly that it is the frame-shifts in the latter two organisms which raise their mean absolute $d$ above the yeast levels. One should therefore conclude that, in yeast, significant disparity is not uncommon either for base-substitutions or for large deletions, and that the amount of disparity in yeast is very similar to that in other fungi when comparable types of mutation are studied.

Disparity or disparity differences could arise in three ways on current recombination models such as that of Meselson and Radding (1975), and the extent of operation of these mechanisms will determine differences in $b$ and $d$. The first, which applies to base-substitutions, frame-shifts and deletions, is by preferential cutting of one of the two DNA strands in h-DNA near a heterozygous site by a repair endonuclease. The second, which only applies to asymmetric h-DNA, is if h-DNA formed preferentially on one type of chromatid (the + allele-bearing one or the $m$ allele-bearing one, in a $+/ m$ heterozygote). The third way is for the frequency of post-meiotic segregation to affect $d$ and $b$. The criterion for classifying mutations into presumed frame-shifts or base-substitutions is that the former tend to have a very low proportion of asci with post-meiotic segregation $(5: 3,3: 5)$ compared to conversion asci with meiotic segregation $(6: 2,2: 6)$, while the proportion of post-meiotic segregations is not nearly so low for presumed base-substitutions (for discussions, see Rossignol, Paquette and Nicolas, 1979; Whitehouse, 1982; Lamb and Ghikas, 1979; Yu-Sun, Wickramaratne and Whitehouse, 1977). If one has only meiotic segregation classes, the most extreme values of $b$ and $d$ are possible, with $b$ of $0.25, d$ of -0.25 when all conversions are to mutant, giving $2: 6$ s only, and $b$ of $0.75, d$ of +0.25 when all conversions are to wild-type, giving $6: 2 \mathrm{~s}$ only. For the post-meiotic segregation classes, less extreme values of $b$ and $d$ are possible, with $b$ of 0.375 and $d$ of -0.125 if all conversions were to mutant, giving $3: 5$ s only, and $b$ of $0.625, d$ of $+0 \cdot 125$, if all conversions were to wild-type, giving $5: 3 \mathrm{~s}$ only. If conversion is very strongly biased in one direction, then frame-shifts will have mainly $6: 2 \mathrm{~s}$ or mainly $2: 6 \mathrm{~s}$, with extreme $b$ and $d$ values, but base-substitutions will usually have mainly $6: 2 \mathrm{~s}$ and $5: 3 \mathrm{~s}$, or mainly $2: 6 \mathrm{~s}$ and $3: 5 \mathrm{~s}$, with less extreme values of $b$ and $d$. Therefore increasing proportions of $5: 3$ and $3: 5$ asci, compared to $6: 2$ and $2: 6$ asci, reduce the range of possible $b$ values and reduce disparity measured by $d$.

For explaining the lower disparities observed for base-substitutions compared with frame-shifts, the third explanation is sufficient on its own. The second explanation seems unlikely because it provides no obvious molecular reason for disparity differences between frame-shifts and basesubstitutions, and also only applies to asymmetric h-DNA. The first explanation above, different extents of preferential strand-cutting, could explain these disparity differences if a repair endonuclease showed greater discrimination between the looped-out and non-looped-out strands for non-pairs in h-DNA for a heterozygous frame-shift than between the two strands in a mispair in h-DNA for a heterozygous base-substitution: this is plausible at the molecular level. If it were true, one should see greater departures 


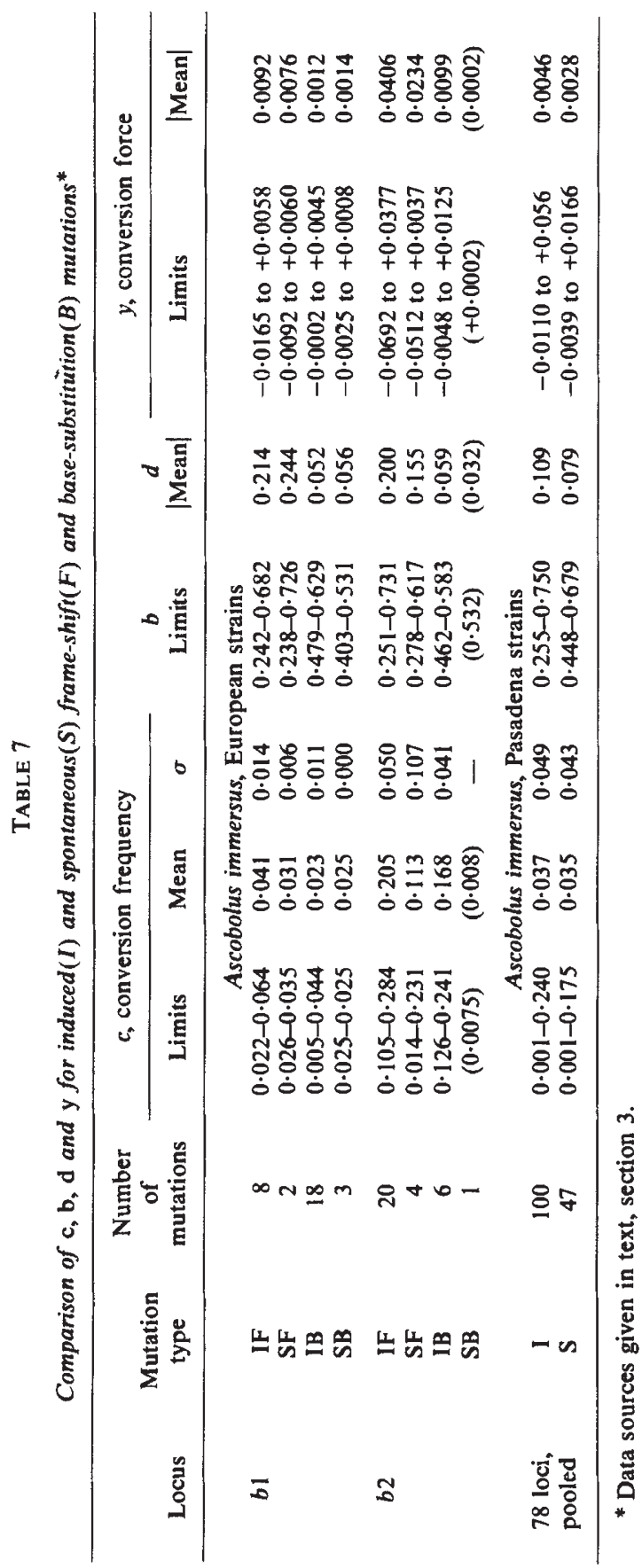


from equality in the $6: 2 / 2: 6$ ratio for frame-shifts than for base-substitutions. This is certainly the case for the $S$. brevicollis data of Yu-Sun, Wickramaratne and Whitehouse (1977), and much of the A. immersus data of Leblon (1972) and Rossignol, Paquette and Nicolas (1979), though with some exceptions; it is less apparent in the Ascobolus data of Lamb and Ghikas (1979) and Lamb and Helmi (1978). It also applies to the non-pairs for long deletions in yeast (see end of section 5(ii)). The 5:3/3:5 departures from equality for base-substitutions are also generally less than for $6: 2 / 2: 6$ for frame-shifts, being more like those for $6: 2 / 2: 6$ for the base-substitutions, although grey -5 in $S$. brevicollis seems an exception; there are many cases of single mutations with different $6: 2 / 2: 6$ and $5: 3 / 3: 5$ disparities in the data referred to.

It seems then that the disparity differences between frame-shifts and base-substitutions can have two separate causes, one relating to more extreme differences in correction direction for frame-shifts, and one relating to frame-shifts having fewer post-meiotic segregations, when post-meiotic segregations can not give such extremities of $b$ and $d$ as can meiotic segregations in conversion asci.

The very large differences found in $y$ for different mutations follow directly from their difference in $c, b$ and $d$. The dispersed distributions of $y$ for different mutations at a locus, with a relatively high proportion of mutations towards the extremes, make it likely that conversion is a significant force in populations and their evolution, though the extent of that significance obviously depends on how general the present results are. The interactions of $y$ values with other factors, especially dominance, selection and mutation, were investigated by Lamb and Helmi (1982). Their findings (e.g., their table 4) showed that even with $c$ values in the Drosophila melanogaster range, conversion could have large effects on equilibrium allele frequencies for selectively neutral genes and for mildly deleterious recessive alleles and alleles with no dominance. For example, under some conditions even changes in $y$ at the sixth or seventh decimal place can have substantial effects on equilibrium allele frequency.

For applications to natural populations, it is important to compare $y$ values for spontaneous and induced mutations. Although larger samples are desirable, the most suitable data are the $A$. immersus results in table 7 . For the $b 1$ locus, differences between spontaneous and induced mutations were slight for $c$, for limits of $b$, and for mean absolute $d$ and $y$. For $b 2, c$ and mean absolute $d$ and $y$ were somewhat lower for spontaneous than for induced mutations. For pooled data in the Pasadena strains, there is little difference in mean $c$, but mean absolute $d$ and $y$ were lower for spontaneous than for induced mutations. From the previous discussion, the expectations of differences between spontaneous and induced mutations for $b, d$ and $y$ depend on the proportions of frame-shifts and base-substitutions amongst them, which depend on the extent to which frame-shift inducing and base-substitution inducing mutagens are used. Taking the $b 1, b 2$ and Pasadena data together, mean absolute values of $y$ for spontaneous mutations are either very similar to those of induced mutations, or slightly less, by a factor of about 1.6 to 1.7. Conclusions about the effects of gene conversion on populations are therefore only influenced slightly by whether the data are for spontaneous mutations, induced mutations, or for both types. 


\section{REFERENCES}

AHMAD, A. F. 1975. The effects of temperature on aberrant ascus frequencies at the Buff locus in Sordaria brevicollis. Genet. Res., 26, 127-135.

BARANOWSKA, H. 1970. Intragenic recombination pattern within the 164 locus of Ascobolus immersus in the presence of outside markers. Genet. Res., 16, 185-206.

BOONE, D. M. AND KEITT, G. W. 1956. Venturia inequalis (Cke). Wint. VIII. Inheritance of color mutant characters. Amer. Jnl. Bot., 43, 226-233.

BRIDGES, C. B. AND OLBRYCHT, T. M. 1926. The multiple stock "Xple" and its use. Genetics, $11,41-56$.

CARLSON, P. S. 1971. A genetic analysis of the rudimentary locus of Drosophila melanogaster, Genet. Res., 17, 53-81.

CATCHESIDE, D. G. 1977. The Genetics of Recombination. Edward Arnold.

DOVER, G. A. 1982. Molecular drive: A cohesive mode of species evolution. Nature, 299, $111-117$.

Dover, G. A., brown, S., COEN, E., DAllas, J., Strachan, T. AND TRiCk, M. 1982. The dynamics of genome evolution and species differentiation. In Dover, G. A. and Flavell, R. B. (eds.) Genome Organisation, Academic Press, pp. 343-372.

FINCHAM, J. R. S., DAY, P. R. AND RADFORD, A. 1979. Fungal Genetics. Blackwell.

FINK, G. R. AND STYLES, C. A. 1974. Gene conversion of deletions in the HIS 4 region of yeast. Genetics, 77, 231-244.

FOGEL, S., HURST, D. D. AND MORTIMER, R. K. 1971. Gene conversion in unselected tetrads from multipoint crosses. In Kimber, G. and Rédei, G. P. (eds.) Stadler Genetics Symposia, vol. 1 and 2, Columbia, pp. 89-110.

FOGEL, S., MORTIMER, R. K., LUSNAK, K. AND TAVARES, K. 1979. Meiotic gene conversion-a signal of the basic recombination event in yeast. Cold Spring Harb. Symp. Quant. Biol., 43, 1325-1341.

FREELING, M. 1978. Allelic variations at the level of intragenic recombination. Genetics, 89, 211-224.

Gajewski, W., PAszewski, A., DAWIdowiCZ, A. AND DUdzinskA, B. 1968. Postmeiotic segregation in locus '46' of Ascobolus immersus. Genet. Res., 11, 311-317.

GIRARD, J. AND ROSSIGNOL, J.-L. 1974. The suppression of gene conversion and intragenic crossing over in Ascobolus immersus: evidence for modifiers acting in the heterozygous state. Genetics, 76, 221-243.

GUTZ, H. 1971. Site specific induction of gene conversion in Schizosaccharomyces pombe. Genetics, 69, 317-337.

GUTZ, H. AND LESLIE, J. F. 1976. Gene conversion: a hitherto overlooked parameter in population genetics. Genetics, $83,861-866$.

HELMI, S. AND LAMB, B. C. 1981. The identification and properties of conversion control factors 2, 3 and 4 affecting a single locus in the Pasadena strains of Ascobolus immersus. Heredity, 46, 289.

HELMI, S. AND LAMB, B. C. 1983. The interactions of three widely separated loci controlling conversion properties of $w$ locus I in Ascobolus immersus. Genetics, 104, 23-40.

HILLIKER, A. J. AND CHOVNICK, A. 1981. Further observations on intragenic recombination in Drosophila melanogaster. Genet. Res., 38, 281-296.

HOWELL, W. M. 1982. The identification and characterization of further genetic factors controlling gene conversion in Ascobolus immersus. Ph.D. Thesis, Imperial College, London.

KITANI, Y. 1982. Characteristics of gene conversion and Rec factor of the $i$ locus of Sordaria fimicola. Jpn. J. Genet., 57, 467-481.

KITANI, Y. AND OLIVE, L. S. 1967. Genetics of Sordaria fimicola. VI. Gene conversion at the $g$ locus in mutant $\times$ wild-type crosses. Genetics, $57,767-782$.

KLEIN, H. L. AND PETES, T. D. 1981. Intrachromosomal gene conversion in yeast. Nature, 289, $144-148$.

KRUSZEWSKA, A. AND GAJEWSKI, W. 1967. Recombination within the Y locus in Ascobolus immersus. Genet. Res., 9, 159-177.

LAMB, B. C. 1969. Related and unrelated changes in conversion and recombination frequencies with temperature in Sordaria fimicola, and their relevance to hybrid-DNA models of recombination. Genetics, 62, 67-78.

LAMB, B. C. 1975. Cryptic mutations: their predicted biochemical basis, frequencies and effects on gene conversion. Molec. Gen. Genet., 137, 305-314.

LAMB, B. C. 1981. Regular and aberrant segregation at meiosis. In Gull, K. and Oliver, S. G. (eds.) The Fungal Nucleus, Cambridge U.P., pp. 215-237. 
LAMB, B. C. AND GHIKAS, A. 1979. The intergradation, genetic interchangeability and interpretation of gene conversion spectrum types. Genetics, 92, 49-65.

LAMB, B. C. AND HELMI, S. 1978. A new type of genetic control of gene conversion, from Ascobolus immersus. Genet. Res., 32, 67-78.

LAMB, B. C. AND HELMI, S. 1982. The extent to which gene conversion can change allele frequencies in populations. Genet. Res., 39, 199-217.

LAWRENCE, C. W., SHERMAN, F., JACKSON, M. AND GILMORE, R. A. 1975. Mapping and gene conversion studies with the structural gene for iso-1-cytochrome $c$ in yeast. Genetics, 81 , 615-629.

LEBLON, G. 1972. Mechanism of gene conversion in Ascobolus immersus. I. Existence of a correlation between the origin of mutants induced by different mutagens and their conversion spectrum. Molec. Gen. Genet., 115, 36-48.

MACDONALD, M. V. AND WHITEHOUSE, H. L. K. 1979. A buff spore colour mutant in Sordaria brevicollis showing high-frequency conversion. I. Characteristics of the mutant. Genet. Res., 34, 87-119.

MESELSON, M. S. AND RADDING, C. M. 1975. A general model for genetic recombination. Proc. Natl. Acad. Sci. U.S., 72, 358-361.

MIKUS, M. D. AND PETES, T. D. 1982. Recombination between genes located on nonhomologous chromosomes in Saccharomyces cerevisiae. Genetics, 101, 369-404.

mousSEAU, J. 1966. Sur les variations de fréquence de conversion au niveau de divers sites d'un même locus. C.R. Acad. Sci. Paris, D, 262, $1254-1257$.

NELSON, O. E. 1962. The waxy locus in maize. I. Intralocus recombination frequency estimafes by pollen and by conventional analyses. Genetics, 47, 737-742.

NELSON, O. E. 1975. The waxy locus in maize. III. Effect of structural heterozygosity on intragenic recombination and flanking marker assortment. Genetics, 79, 31-44.

PASZEWSKi, A. AND PRAZMO, W. 1969. The bearing of mutant and cross specificity on the pattern of intragenic recombination. Genet. Res., 14, 33-43.

ROSSIGNOL, J.L. 1969. Existence of homogeneous categories of mutants exhibiting various conversion patterns in gene 75 of Ascobolus immersus. Genetics, 63, 795-805.

RosSignol, J-L., PAQUETTE, N. AND NICOLAS, A. 1979. Aberrant 4:4 asci, disparity in the direction of conversion, and frequencies of conversion in Ascobolus immersus. Cold Spring Harb. Symp. Quant. Biol., 43, 1343-1352.

SANG, H. AND WHITEHOUSE, H. L. K. 1979. Genetic recombination at the buff spore colour locus in Sordaria brevicollis. Molec. Gen. Genet., 174, 327-334.

SMITH, P. D., FINNERTY, V. G. AND CHOVNICK, A. 1970. Gene conversion in Drosophila: non-reciprocal events at the maroon-like cistron. Nature, 228, 442-444.

TOURÉ, B. 1972. Double reversal of gene conversion polarity and multiple conversion events in the locus "14" in Podospora anserina. Molec. Gen. Genet., 117, 267-280.

WHITEHOUSE, H. L. K. 1974. Aberrant ascus genotypes from crosses involving mutants at the $\mathrm{g}$ locus in Sordaria fimicola. Genet. Res., 24, 229-250.

WhITEHOUSE, H. L. K. 1982. Genetic Recombination. Wiley.

WICKRAMARATNE, M. R. T. AND LAMB, B. C. 1978. The estimation of conversion parameters and the control of conversion in Ascobolus immersus. Molec. Gen. Genet., 159, 63-73.

YU.SUN, C. C. C. 1966. Linkage groups in Ascobolus immersus. Genetica, 37, 569-580.

YU-SUN, C. C. C., WICKRAMARATNE, M. R. T. AND WHITEHOUSE, H. L. K. 1977. Mutagen specificity in conversion pattern in Sordaria brevicollis. Genet. Res., 29, 65-81. 